\title{
FGF10 and Lipofibroblasts in Lung Homeostasis and Disease: Insights Gained From the Adipocytes
}

\begin{abstract}
Yu-Qing Lv ${ }^{1,2+}$, Qhaweni Dhlamini ${ }^{2 \dagger}$, Chengshui Chen ${ }^{1}$, Xiaokun $L^{2}{ }^{2}$, Saverio Bellusci ${ }^{1,3 *}$ and Jin-San Zhang ${ }^{1,2 *}$

'Key Laboratory of Interventional Pulmonology of Zhejiang Province, Center for Precision Medicine, The First Affiliated Hospital of Wenzhou Medical University, Wenzhou, China, ${ }^{2}$ International Collaborative Center on Growth Factor Research, School of Pharmaceutical Sciences, Wenzhou Medical University, Wenzhou, China, ${ }^{3}$ Cardio-Pulmonary Institute, Institute of Lung Health and Department of Pulmonary and Critical Care Medicine and Infectious Diseases, Universities of Giessen and Marburg Lung Center (UGMLC), Member of the German Center for Lung Research (DZL), Justus-Liebig University Giessen, Giessen, Germany
\end{abstract}

\section{OPEN ACCESS \\ Edited by: \\ Ming Li, \\ Osaka University, Japan}

Reviewed by:

Siegfried Ussar,

Helmholtz-Gemeinschaft

Deutscher Forschungszentren ( $\mathrm{HZ})$,

Germany

Jiqiu Wang,

Shanghai Jiao Tong University, China

${ }^{*}$ Correspondence:

Saverio Bellusci

saverio.bellusci@innere.med.

uni-giessen.de

Jin-San Zhang

zhang_jinsan@wmu.edu.cn

these authors have contributed equally to this work

Specialty section:

This article was submitted to

Stem Cell Research,

a section of the journal

Frontiers in Cell and Developmental

Biology

Received: 23 December 2020

Accepted: 28 April 2021

Published: 26 May 2021

Citation:

Lv Y-Q, Dhlamini Q, Chen C, LiX, Bellusci S and Zhang J-S (2021) FGF10 and Lipofibroblasts in Lung Homeostasis and Disease: Insights Gained From the Adipocytes. Front. Cell Dev. Biol. 9:645400. doi: 10.3389/fcell.2021.645400
Adipocytes not only function as energy depots but also secrete numerous adipokines that regulate multiple metabolic processes, including lipid homeostasis. Dysregulation of lipid homeostasis, which often leads to adipocyte hypertrophy and/or ectopic lipid deposition in non-adipocyte cells such as muscle and liver, is linked to the development of insulin resistance. Similarly, an altered secretion profile of adipokines or imbalance between calorie intake and energy expenditure is associated with obesity, among other related metabolic disorders. In lungs, lipid-laden adipocyte-like cells known as lipofibroblasts share numerous developmental and functional similarities with adipocytes, and similarly influence alveolar lipid homeostasis by facilitating pulmonary surfactant production. Unsurprisingly, disruption in alveolar lipid homeostasis may propagate several chronic inflammatory disorders of the lung. Given the numerous similarities between the two cell types, dissecting the molecular mechanisms underlying adipocyte development and function will offer valuable insights that may be applied to, at least, some aspects of lipofibroblast biology in normal and diseased lungs. FGF10, a major ligand for FGFR2b, is a multifunctional growth factor that is indispensable for several biological processes, including development of various organs and tissues such as the lung and WAT. Moreover, accumulating evidence strongly implicates FGF10 in several key aspects of adipogenesis as well as lipofibroblast formation and maintenance, and as a potential player in adipocyte metabolism. This review summarizes our current understanding of the role of FGF10 in adipocytes, while attempting to derive insights on the existing literature and extrapolate the knowledge to pulmonary lipofibroblasts.

Keywords: FGF10, adipocytes, adipocyte-like cells, stem cell, lipofibroblast, myofibroblast, lung regeneration/repair

\section{INTRODUCTION}

FGF10, a member of the FGF family, is a potent mitogen that is indispensable for proper development, regeneration, and health. In general, FGFs elicit biological responses by binding to and activating four highly conserved transmembrane tyrosine kinase receptors (FGFR1-4). Currently, the FGF family comprises at least 22 members that are classified into seven subfamilies 
based on sequence homology, functional properties, and evolutionary phylogeny. FGF10 belongs to the FGF7 subfamily, a group consisting of FGF3, 7, 10, and 22 (Ornitz and Itoh, 2015), and binds with higher affinity to FGFR1b and FGFR2b compared to the other FGF receptors (Bellusci et al., 1997). More details regarding FGF10, including its protein structure and function, expression profile in tissues or during development, and signaling transduction mechanisms are comprehensively reviewed elsewhere (Ndlovu et al., 2018). Among the FGFs, Fgf10 is well-characterized for its roles in development of various organs and tissues, including the lung and white adipose tissue (WAT) (Gartside et al., 2009; Ohta and Itoh, 2014; Ornitz and Itoh, 2015). Mice lacking Fgf10 or its primary receptor Fgfr2b display multiple organ defects, including complete lung agenesis (Min et al., 1998; De Moerlooze et al., 2000; Ohuchi et al., 2000). FGF10 expression was observed in adipose tissue and has been strongly implicated in adipogenesis (Sakaue et al., 2002; Patel et al., 2005; Ohta and Itoh, 2014). Significantly, Fgf10 knockout (KO) mice display impaired WAT development, indicating that Fgf10 expression is crucial for normal WAT development (Yamasaki et al., 1999; Sakaue et al., 2002). Herein, we summarize the current knowledge and gaps in our understanding of the role of FGF10 in adipocytes and adipocytelike cells.

\section{SUCCINCT BACKGROUND ON ADIPOSE TISSUE AND ADIPOGENESIS}

The adipose tissue (AT) is a dynamic metabolic and endocrine organ that contributes to various crucial physiological processes, including regulation of energy balance and metabolic homeostasis. In mammals, AT is classified into two morphologically and functionally distinct types: WAT and brown adipose tissue (BAT) (Rosen and Spiegelman, 2000). WAT consists generally of spherical cells that contain a large uniocular lipid droplet occupying the majority of the cytosol and markedly lower mitochondria content than brown adipocytes (Cannon and Nedergaard, 2004; Cinti, 2005). WAT primarily stores excess energy in the form of triglycerides and mobilizes the energy depending on the state of energy balance and immediate physiological needs. Furthermore, WAT secretes several endocrine factors, such as adipokines and cytokines, that act on various targets to regulate multiple metabolic processes (Ahima and Lazar, 2008). BAT, in contrast to WAT, consists of ellipsoidal cells that contain multiple, small, multilocular lipid droplets, and higher content of UCP1-expressing mitochondria (Cannon and Nedergaard, 2004; Cinti, 2005). BAT mainly dissipates chemical energy via UCP1-mediated mitochondrial uncoupling, generating heat in the process; a phenomenon often dubbed "non-shivering thermogenesis" (Lowell and Flier, 1997; Cannon and Nedergaard, 2004; Richard and Picard, 2011).

Adipogenesis is a multi-step process by which mature adipocytes arise from mesenchymal stem cells (MSCs) through cell differentiation. This process is regulated by a complex network of transcription factors in concert with several extracellular mediators, such as hormones and growth factors, and occurs in two major phases. First, MSCs commit to preadipocytes, and second, preadipocytes terminally differentiate into mature, functional adipocytes (Rosen and Macdougald, 2006). Adipocytes represent the primary building blocks of adipose tissue, and three types of adipocytes deriving from distinct MSC lineages constitute two main types of adipose tissue. White adipocytes mainly derive from the Myf5-negative lineage, while brown adipocytes arise from the Myf5-positive lineage. Some studies, however, indicate that Myf5-positive precursors may give rise to a subset of white adipocytes as well (Sanchez-Gurmaches and Guertin, 2014; Sebo et al., 2018). In addition to classical brown adipocytes, a distinct type of thermogenic, brown adipocyte-like cells known as Beige adipocytes have been found in WAT depots (Cannon and Nedergaard, 2004; Wu et al., 2012). Beige adipocytes are thought to arise from: (a) mature white adipocytes via $\beta 3$ adrenergic stimulation, chronic Peroxisome proliferator-activated receptor gamma (PPAR- $\gamma$ ) induction, and cold exposure-induced transdifferentiation (Barbatelli et al., 2010), (b) platelet-derived growth factor receptor $\alpha$ (PDGFR $\alpha)$ positive white preadipocytes via differentiation (Figure 1; Fischer et al., 2017), and (c) Myf5 negative MSCs (Sanchez-Gurmaches et al., 2016). Beige and brown adipocytes share several biochemical characteristics, specifically, they both contain abundant UCP1-expressing mitochondria and multilocular lipid droplets.

\section{ROLE OF FGF10 IN ADIPOGENESIS AND WAT BROWNING}

Autocrine/paracrine FGF10 signaling has been implicated in WAT development, remodeling, and metabolism (Ohta and Itoh, 2014). FGF10 is shown to play a crucial role in the differentiation of preadipocytes in WAT via activation of PPAR$\gamma$, the key transcriptional regulator of adipogenesis (Sakaue et al., 2002; Asaki et al., 2004). KO mice studies revealed that loss of Fgf10 expression in embryonic WAT results in markedly reduced expression of $P P A R-\gamma$, indicating that FGF10 acts upstream of $P P A R-\gamma$ to stimulate adipogenesis (Ohta et al., 2011). Furthermore, blockade of Fgf10 signaling inhibited the expression of $\mathrm{C} / \mathrm{EBP} \alpha$, a major adipogenic transcription factor that is critical for the initiation of 3T3-L1 cell differentiation, thereby suppressing adipocyte development in differentiating 3T3-L1 preadipocytes (Lane et al., 1999; Sakaue et al., 2002). These data suggest that FGF10 expression or activity is critical for preadipocyte differentiation into mature adipocytes. Although FGF10 is shown to promote adipogenesis/terminal adipocyte differentiation via upregulation of the adipogenic genes PPAR$\gamma, \mathrm{C} / \mathrm{EBP} \alpha$, and lipoprotein lipase (LPL), it remains unclear whether FGF10 may induce/regulate adipogenic cell fate determination in undifferentiated MSCs, as most, if not all studies investigating the role of Fgf10 in adipogenesis have employed the 3 T3-L1 cell line, which is already committed to adipocyte lineage. Thus, further studies employing multipotent undifferentiated MSC cell lines, are warranted to investigate the potential effects of FGF10 on adipogenic cell fate commitment of MSCs in vivo. 


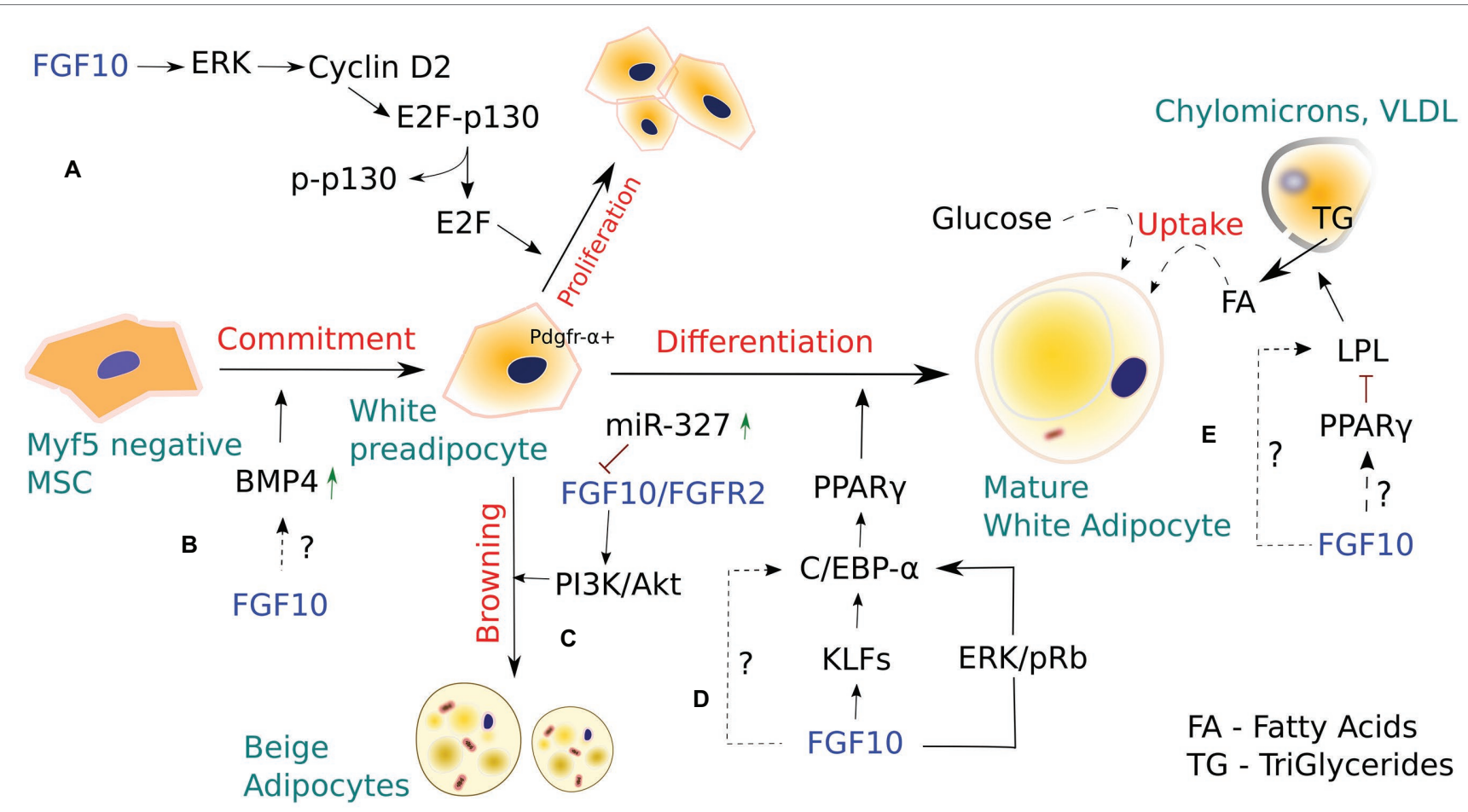

FIGURE 1 | The role of FGF10 in white and Beige adipocyte development. (A) FGF10-stimulated activation of the Ras/MAPK pathway induces proliferation of white preadipocytes via cyclin D2-dependent phosphorylation of p130. (B) Bone morphogenic protein 4 (BMP4), acting downstream of FGF10, is known to regulate FGF10-mediated bud outgrowth during branching morphogenesis of the developing lung, thus contributing to controlling bud size. In adipogenesis, BMP4 stimulates commitment of pluripotent stem cells toward the white adipocyte lineage. However, whether a dynamic interplay between FGF10 and BMP4 occurs in adipogenesis, similar to that seen in embryonic lung development, remains to be elucidated. (C) FGF10 is part of a miRNA-327-regulated autocrine loop that stimulates development of Beige adipocytes from Pdgfr $\alpha+$ white preadipocytes. However, Beige adipocytes are also thought to arise from mature white adipocytes via $\beta$-3 adrenergic stimulation, cold exposure, and chronic PPAR- $\gamma$ induction. (D) FGF10, via a number of signaling routes, activates downstream PPAR- $\gamma$, which in turn induces white adipocyte differentiation. (E) FGF10 overexpression in intramuscular adipocytes enhanced the expression of lipoprotein lipase (LPL) and LPLinduced lipid accumulation, suggesting a possible role for FGF10 in regulating adipocyte metabolism. However, LPL is also known to be regulated by PPAR- $\gamma$, and thus whether FGF10 would target LPL directly or via PPAR- $\gamma$ to exert its effects in adipocyte metabolism remains to be determined.

FGF10 is abundantly expressed in the Stromal Vascular Fraction of WAT, which is comprised of adipocyte stem and progenitors among other cell types, suggesting that FGF10 is essential for adipogenesis in vivo (Yamasaki et al., 1999). Classical observations indicate that FGF10 expression in WAT stimulates both proliferation and differentiation of preadipocytes via the Ras/MAPK pathway (Figure 1; Asaki et al., 2004; Konishi et al., 2006; Ohta et al., 2011). In preadipocytes, FGF10/FGFR2b signaling via the Ras/MAPK cascade was shown to trigger $\mathrm{pRb}$ and subsequent $\mathrm{Rb}-\mathrm{C} / \mathrm{EBP}$ complex formation, leading to downstream activation of $P P A R-\gamma$, which in turn induces adipogenesis. FGF10 was also shown to mediate preadipocyte proliferation in WAT by inducing cyclin D2-dependent phosphorylation of P130 through the Ras/MAPK pathway. In support of these observations, Fgf10 KO embryos exhibited aberrant Cyclin D2 expression and P130 phosphorylation in the WAT and markedly impaired preadipocyte proliferation (Konishi et al., 2006; Ohta et al., 2011).

In addition, specific blockade of Fgf10/Fgfr2 and downstream AKT signaling with miR-327, a key regulator of preadipocyte differentiation into Beige adipocytes, resulted in impaired preadipocyte differentiation, indicating that Fgf10 may indeed drive preadipocyte differentiation via the Fgfr2-Akt axis. Furthermore, miR-327 was shown to regulate Fgf10-mediated preadipocyte differentiation by inhibiting the Fgf10-activated Fgfr2-Akt signaling cascade (Fischer et al., 2017). However, using the established mouse 3T3-L1 cell line to investigate the effect of Fgf10 and $m i R-327$ levels on preadipocyte proliferation, the researchers demonstrated that Fgf10 expression levels do not affect preadipocyte proliferation (Fischer et al., 2017).

Fischer et al. (2017) reported the existence of a miRNA327-FGF10-FGFR2 autocrine regulatory loop in PDGFR $\alpha+$ cells, which is critical for WAT browning. They demonstrated, for the first time, that a mechanism involving FGF10 could regulate PDGFR $\alpha+$ preadipocyte differentiation into thermogenic Beige adipocytes. These findings are particularly intriguing because PDGFR $\alpha+$ preadipocytes, similar to mesenchymal progenitors of pulmonary lipofibroblasts (LIFs), not only differentiate via a mechanism involving Fgf10 upregulation but also express a number of shared adipogenic genes. Considering the similarities between beige and LIF progenitors, we postulate that, similar to beige/white adipocyte- and bipotent PDGFR $\alpha+$ progenitors, LIF progenitors, via Fgf10 upregulation, may also give rise to 
distinct LIF cell subtypes that may play some as yet undiscovered roles in lung biology.

\section{OTHER FGF FAMILY MEMBERS ARE IMPLICATED IN ADIPOSE TISSUE DEVELOPMENT, REMODELING, AND FUNCTION}

Apart from FGF10, other FGF family members, including FGF21 and FGF9, play crucial roles as autocrine/paracrine adipokines in regulating energy homeostasis by influencing WAT or BAT/beige development, remodeling, and function. FGF21 has been shown to activate thermogenesis via induction of WAT browning and thermogenic genes, such as PGC1 and UCP1, following cold exposure (Fisher et al., 2012). FGF21 KO mice exhibited an impaired adaption to chronic cold exposure and diminished WA browning, suggesting that FGF21 is critical for normal adaptations to cold exposure. In addition, FGF21 via a feed-forward loop involving PPAR- $\gamma$, regulates insulin sensitivity and glucose homeostasis in WAT (Dutchak et al., 2012), suggesting that FGF21 contributes to the antidiabetic activities of PPAR- $\gamma$. On the contrary, FGF9 upregulation was shown to negatively regulate WAT browning and the expression of thermogenic genes, including PGCland UCP1, and positively correlate with obesity (Sun et al., 2019). The inhibitory effects of FGF9 on WAT browning were accompanied with decreased expression of adipogenic markers including $C / E B P \beta$ and $P P A R-\gamma$, indicating that FGF9 upregulation inhibits adipogenesis. Besides FGF21 and FGF9, other FGFs such as FGF1, FGF16, and FGF19, also have important roles in adipogenesis; however, due to space limitations, these growth factors will not be covered here. Taken together, these findings provide new insights for FGFs as potential targets for treating obesity and its related metabolic disorders.

\section{FGF10 IN THE REGULATION OF MULTIPLE ADIPOCYTE METABOLIC FACTORS}

\section{Lipoprotein Lipase}

Lipoprotein lipase, a member of the lipase superfamily, is chiefly expressed in tissues that oxidize or store large amounts of fatty acids, such as WAT and BAT, and is an important early marker of adipocyte differentiation (Amri et al., 1996). LPL hydrolyzes triglycerides from circulating triglyceriderich lipoproteins (VLDL and chylomicrons) into fatty acids, which are then taken up by adipocytes for storage or energy production via beta-oxidation (Mead et al., 2002). Specific siRNA-mediated knockdown of $F g f 10$ in goat intramuscular preadipocytes inhibited lipid droplet accumulation, and impaired adipocyte development, suggesting that Fgf10 plays a pivotal role in adipocyte development and metabolism, as lipid accumulation represents a major functional characteristic of adipocytes (Xu et al., 2018). Beyond its adipogenesis activity, PPAR- $\gamma$ is involved in various aspects of adipocyte lipid metabolism and regulates the expression of adipocyte-specific genes associated with lipid accumulation and metabolism, including LPL (Schoonjans et al., 1996; Kersten et al., 2000). Interestingly, Fgf10 overexpression in intramuscular adipocytes enhanced the expression of LPL among other transcription factors, while Fgf10 knockdown using RNA interference suppressed the expression of LPL and LPL-induced lipid accumulation (Xu et al., 2018). These findings suggest a possible role for FGF10 in regulating adipocyte metabolism while raising the question of whether FGF10 would target LPL directly or via PPAR- $\gamma$ to exert its effects in adipocyte metabolism.

\section{Kruppel-Like Factors}

Kruppel-like factors (KLFs) are a large family of zinc-finger proteins with functionally diverse roles in various physiological and cellular processes, including adipogenesis (Pearson et al., 2008; Pei et al., 2011). KLFs constitute the central transcriptional cascade that acts sequentially and upstream of the adipogenic master regulator PPAR- $\gamma$ to negatively or positively regulate adipogenesis (Wu and Wang, 2013). Recently, Fgf10 was shown to upregulate mRNA expression of KLF3, 9, and 13 in goat intramuscular preadipocytes, indicating a possibility that Fgf10 may regulate adipocyte development, at least in part, by targeting KLF3, 9, and 13 (Xu et al., 2018). However, further studies are needed to clarify the precise effects of these KLFs on adipogenesis, and to establish the specific mechanisms involved (Xu et al., 2018).

\section{Bone Morphogenic Proteins}

Bone morphogenic proteins (BMPs) are known to play crucial roles in several hallmarks of adipogenesis, including adipocyte lineage commitment, preadipocyte differentiation, and support of adipocyte function (Fux et al., 2004; Huang et al., 2009; Schulz et al., 2011). BMPs are polypeptide growth factors that belong to the TGF- $\beta$ superfamily and are involved in several aspects of embryonic lung patterning and adipogenesis. Among the BMP family members, BMP2, BMP4, and BMP7 are the most studied factors for their roles in adipogenesis. BMP2 and BMP4 are essential for white adipocyte development (Fux et al., 2004; Huang et al., 2009), while BMP7 is involved in brown adipocyte development (Schulz et al., 2011). BMP4 plays a critical role in adipogenesis and has been shown not only to stimulate commitment of pluripotent stem cells toward the white adipocyte lineage but also promote WAT differentiation (Otto et al., 2007). Interestingly, BMP4 was identified to act in concert with FGF10 during lung development. BMP4, acting downstream of FGF10, regulates branching and patterning of the developing lung, thus contributing to bud size control. During branching morphogenesis, FGF10 induces bud formation in proximal-distal regions and subsequent epithelial expression of BMP4, which in turn limits further FGF10-mediated bud outgrowth (Weaver et al., 2000). Given that both FGF10 and BMP4 have been implicated in 
adipogenesis, it will be interesting to study whether a dynamic interplay between FGF10 and BMP4 occurs in adipogenesis, for instance, as similar to that seen in embryonic lung development.

\section{THE ROLES OF FGF10 IN ADIPOCYTES MAY BE EXTRAPOLATED TO LIPOFIBROBLASTS}

Lipofibroblasts and adipocytes share several common characteristics, despite their distinct anatomical locations. For one thing, both LIFs and adipocytes are lipid-laden cells that characteristically express an array of adipogenic genes and markers, such as Adrp, PPAR- $\gamma$, and LPL, associated with various aspects of their biological processes, including lipid metabolism and lipogenesis. The roles of some of these markers in LIF and adipocyte cellular biology are discussed in detail in other sections of this review. Furthermore, LIFs and adipocytes utilize shared developmental signaling pathways during their formation, including Fgf10, PPAR- $\gamma$, and PDGFR $\alpha$ signaling. Both cell types emerge from MSCs via Fgf10-mediated differentiation of their mesenchymal progenitors into mature cells (Ohta et al., 2011; Al Alam et al., 2015). In addition, like lipofibroblasts, adipocytes require $P P A R-\gamma$ signaling during their maturation and for maintenance of their phenotype. Moreover, apart from FGF10 and PPAR- $\gamma$, the mesenchymal progenitors of both LIFs and adipocytes express PDGFR $\alpha$, whose expression levels progressively decrease during their course of differentiation.

Given the many similarities between adipocytes and LIFs, it is plausible to draw a comparison between the two cells. We, therefore, postulate that the established and potential roles of FGF10 signaling in adipocytes can be extrapolated to pulmonary LIFs. While earlier studies have also predicted the role of Fgf10 in LIF formation by extrapolating from its role in adipogenesis (Al Alam et al., 2015), further studies to test whether, with regard to FGF10 signaling, the similarities between LIFs and adipocytes extend from cellular development to other aspects of their biology. Such studies may help unravel more mysteries of LIF biology and function in lung development, homeostasis, and regeneration.

During embryonic lung development, LIFs originate from several progenitor pools, including a subset of FGF10-expressing cells in the lung mesenchyme (Al Alam et al., 2015). Lineage tracing of Fgf10-positive (FGF10+) cells using Fgf10 knock-in mice revealed that mesenchymal-derived FGF10+ progenitor cells give rise to multiple lineages, including myogenic and adipogenic lineages (El Agha et al., 2014). Furthermore, FGF10 signaling was shown to promote the adipogenic differentiation potential of MSCs through preferential induction of LIF (adipogenic) differentiation rather than alveolar MYF (myogenic) differentiation during lung development (El Agha et al., 2014), suggesting that FGF10 signaling may control early cell fate decisions of MSCs as well as induce conversions between established lineages. The possibility that FGF10 signaling may regulate MSC fate would have profound implications, especially in the context of lung repair and regeneration following injury or disease.

\section{FGF10-FGFR2B SIGNALING IN LIPOFIBROBLASTS - ALVEOLAR TYPE 2 CELLS TWO-WAY COMMUNICATION}

Pulmonary lipofibroblasts were first described five decades ago, and since this initial landmark report, various research groups have invested their efforts to characterize their development, morphology, and their biological roles in vivo. The capacity of LIFs to recruit, store, and supply alveolar type 2 (AT2) cells with lipid substrates for lung surfactant production (Rehan and Torday, 2014), has offered exciting opportunities to make important discoveries that will impact human health.

In vivo tracer studies have shown that LIF cells usually reside next to AT2 cells. Therefore, LIF cells are considered to be essential components of AT2 stem cell niches, and an essential factor in maintaining AT2 cell stemness (Barkauskas et al., 2013; El Agha and Bellusci, 2014; Al Alam et al., 2015). A myriad of studies indicate that AT2 cells and juxtaposed LIFs interact reciprocally via paracrine signaling pathways, such as the PPAR- $\gamma$ pathway, in coordinating normal development, homeostasis, and regeneration/repair of the distal lung (Tordet et al., 1981; El Agha et al., 2014).

Apart from LIFs, FGF10 has been implicated in promoting AT2 lineage formation. Chao et al. (2017) found that lungs of $\mathrm{FgflO}^{+/-}$mice had decreased total number of epithelial cells compared to wild-type mice, and unbalanced alveolar epithelial cell population, with a decreased proportion of AT2 cells and an increased proportion of AT1 cells. Furthermore, FGF10 hypomorphic mice with significantly reduced FGF10 expression levels exhibited marked AT2 defects compared to normal wildtype mice (Chao et al., 2017), further indicating that FGF10 plays a vital role in AT2 lineage formation.

AT2 cells normally play essential roles in host defense, barrier function, and normal lung homeostasis/repair. AT2 cells are generally small, rounded, or cuboidal cells containing characteristic lamellar bodies and apical microvilli (Mason, 2006), and distributed between alveolar epithelial type 1 cells (AT1) in the alveolar septa. In normal adult lungs, AT2 cells are more abundant than AT1 cells, accounting for $14-16 \%$ of all alveolar epithelial cells, and serve as an important progenitor during repair after lung injury and during normal lung homeostasis. AT1 cells appear to be more susceptible to injury from either endogenous or exogenous factors, while AT2 cells are more resistant. In severe cases of lung injury, such as the COVID-19 infection (Chen et al., 2020), the alveolar epithelium usually exhibits widespread AT1 cell necrosis, characterized by a denuded alveolar basement membrane and formation of hyaline membranes, one of the hallmark features of acute respiratory distress syndrome (ARDS).

Following epithelial injury resulting in loss of both AT1 and AT2 cells, surviving AT2 migrate and undergo compensatory proliferation along the alveolar septa, in an attempt to repopulate the denuded epithelial barrier, and eventually transdifferentiate 
to replace lost AT1 cells (Kim et al., 2006). Recent findings, however, suggest that although AT2 cells can contribute to alveolar epithelial regeneration after injury, some of the newly generated alveolar epithelial cells (AECs) may arise from activated Bronchial epithelial stem cells (BESCs; Yuan et al., 2019). In a bleomycin model of lung injury, BESCs function as a source of alveolar epithelial regeneration and repair by differentiating toward AT1 and AT2 cell lineages over of Basal cells (BCs) in honeycomb cysts, in response to increased FGF10 signaling (Yuan et al., 2019). More on these findings will be further discussed in the FGF10 and respiratory disease section. Overall, these observations suggest that elevated FGF10 levels in BESCs may induce efficient epithelial repair via BESCs surrogacy for incapacitated AT2 progenitors in case of severe lung injury (Figure 2A) and that FGF10-based therapies based on this phenomenon may prove beneficial for ARDS and/or COVID-19 patients.

\section{FGF10 IN THE LIF TO MYF SWITCH DURING FIBROSIS FORMATION AND RESOLUTION}

Myofibroblasts (MYF) play an important role in both physiological and pathological repair processes (Kis et al., 2011; Hinz et al., 2012). In physiological repair, myofibroblasts are transiently activated to facilitate wound closure by producing extra cellular matrix (ECM) proteins, notably fibronectin and collagens type
I and III (Tomasek et al., 2002; Klingberg et al., 2013). Successful wound repair is followed by degradation of the provisional matrix and disappearance of myofibroblasts via a number of mechanisms, including apoptosis. In pathological repair, however, myofibroblasts are continuously activated due to ongoing injury, often leading to excessive deposition of aberrant ECM and abnormal tissue repair. Moreover, these persistent myofibroblasts tend to resist apoptosis and, hence, their physiological clearance. Myofibroblast persistence leads to pathological scar formation, and ultimately organ fibrosis.

In pulmonary fibrosis, the origins of activated myofibroblasts remain speculative; however, several sources have been implicated. Recently, lineage-tracing studies implicated FGF10-expressing LIFs as a novel source of the activated MYFs (El Agha et al., 2017b). These studies not only indicated that LIFs transdifferentiate into activated MYFs following bleomycininduced injury but also that a subpopulation of the myofibroblasts reverts to a quiescent phenotype characteristic of the pre-existing LIFs during fibrosis resolution. Unraveling the origins and/or fate of activated myofibroblasts during fibrosis resolution offers the unique opportunity to clearly define pathological hallmarks of lung fibrosis, and discover novel therapeutic interventions that may inhibit or even reverse the switch between lipogenic and myogenic phenotype, and ultimately accelerate fibrosis regression.

Furthermore, activation of PPAR- $\gamma$ signaling via rosiglitazone (a potent PPAR- $\gamma$ agonist) treatment antagonized TGF- $\beta$-mediated myofibroblast differentiation likely by reinforcing the lipogenic

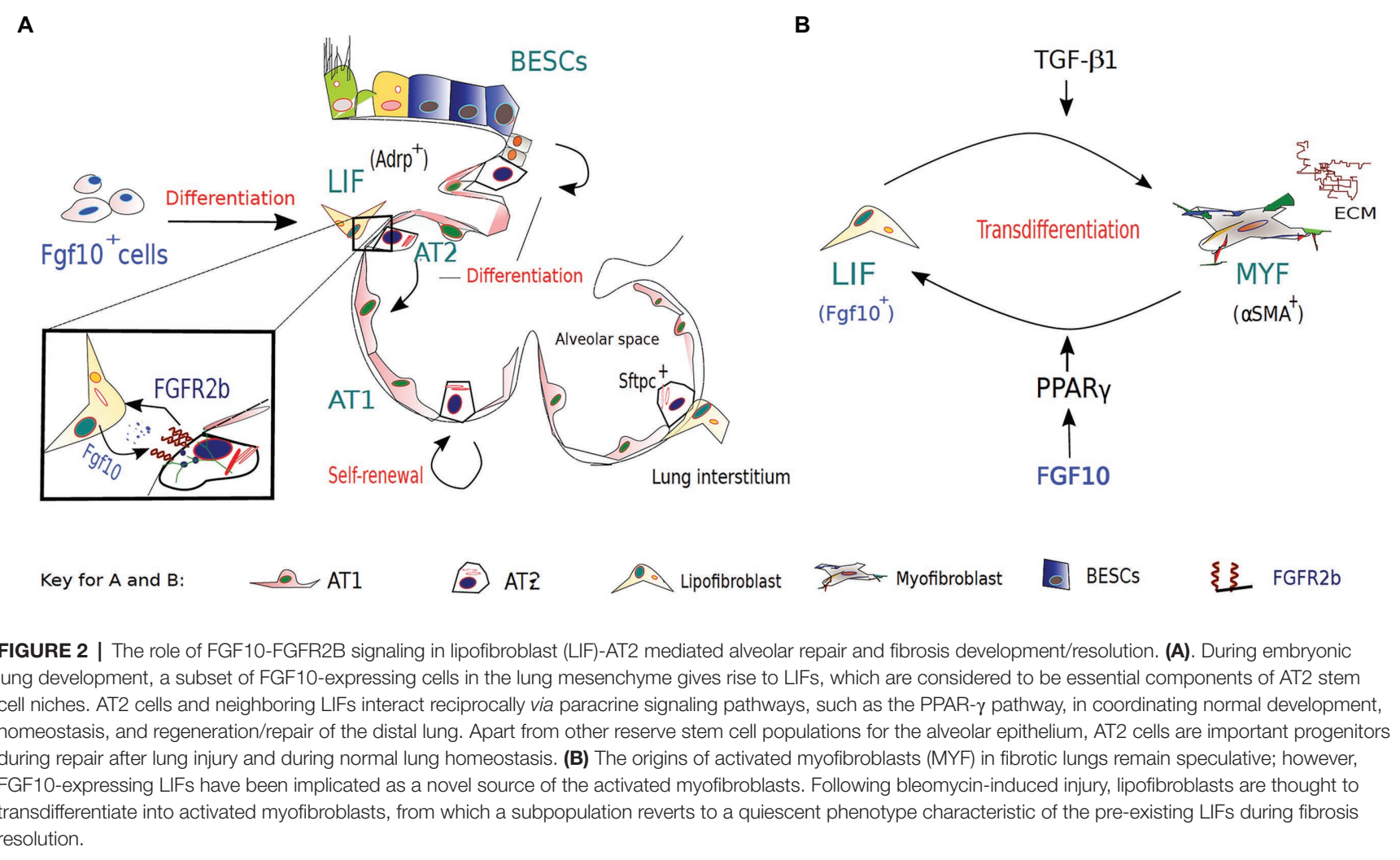


phenotype (El Agha et al., 2017b). Interestingly, TGF- $\beta$ upregulation has been found to be induced by SARS-Cov-2 infection (Agrati et al., 2020; Chen, 2020; Ferreira-Gomes et al., 2021) and associated with several complications of severe COVID19, such as ARDS and pulmonary fibrosis. Thus, it is plausible that FGF10, likely via PPAR- $\gamma$ activation, gets involved in an attempt to replenish LIFs lost to unrestrained MYF differentiation - driven by TGF- $\beta$ upregulation and chronic epithelial injury (Figure 2B). The possibility that FGF10, via $P P A R-\gamma$ signaling, can drive reinforcement of the lipogenic phenotype at the expense of the myogenic phenotype raises the potential that FGF10-based therapies could be beneficial in combating idiopathic pulmonary fibrosis (IPF) and/or COVID-19 related pulmonary fibrosis.

\section{FGF10-FGFR2B SIGNALING AND OTHER ASSOCIATED RESPIRATORY DISEASES}

Aberrant FGF10-FGFR2B signaling contributes to the pathophysiology of multiple respiratory diseases, including IPF and bronchopulmonary dysplasia (BPD). IPF is a chronic age-related respiratory disease of unknown etiology belonging to a heterogeneous group of interstitial lung diseases (ILDs) that are characterized by distorted pulmonary architecture, compromised lung function, and respiratory failure. By way of background, it is worth emphasizing that IPF differs from COVID-19 related pulmonary fibrosis in regard to certain etiological aspects, despite both being characterized by aberrant accumulation of myofibroblasts, reduced lung compliance, and excessive deposition of collagen and other ECM (Sime and O'Reilly, 2001; King et al., 2011; Steele and Schwartz, 2013). More importantly, pulmonary fibrosis is a common consequent manifestation of several acute and chronic ILDs such as ARDS - a major cause of morbidity and mortality in COVID-19 patients (Kruglikov and Scherer, 2020).

Interestingly, apart from fibrotic lungs, reduced lung compliance, as well as AT2 cells with altered surfactant protein expression, have been observed in different models of obesity (Inselman et al., 2004; Foster et al., 2010; Correll et al., 2019). The association of obesity with lung pathologies is gaining much attention, as obesity is increasingly implicated as a major risk factor in several lung disorders, including pulmonary fibrosis and severe COVID-19 infection. In an obese state, SARS-Cov-2 infection is thought to additionally modify the already-compromised adipocytes and adipocyte-like cells by altering their lipid metabolism and promoting their adoption of a differentiation-prone cell state, thus negatively affecting global metabolic homeostasis. In severe cases of SARS-Cov-2 infection, this may involve the lipogenic-to-myogenic switch, a key event in pulmonary fibrogenesis (Kruglikov and Scherer, 2020). Moreover, obese IPF patients have significantly higher waitlist mortality and post-transplant mortality of just over 3 months, compared with controls (Nathan et al., 2010; Gries et al., 2015). In addition, obesity is associated with worse outcome of COVID-19 infection, dysregulated lipid metabolism, and or ectopic lipid accumulation in non-adipocytes, such as pulmonary LIF and neighboring AT2 cells. Therefore, understanding LIF- and or AT2-cell behavior in the context of obesity and COVID-19 may unlock the mysteries associated with COVID-19 pathogenesis.

Dysregulated lipid metabolism in AT2 cells has been linked to an altered surfactant profile (Schmidt et al., 2002; Foster et al., 2010), which, we hypothesize, would increase AT2 cell susceptibility to COVID-19 infection, as surfactant proteins are known to participate in host defense against viral infection (Hsieh et al., 2018). Similarly, ectopic accumulation of lipid in AT2 cells has been shown to induce AT2 hyperplasia (Foster et al., 2010), which, we again hypothesize, may translate to more ACE2-expressing AT2 cells - the primary target of the COVID-19 virus (Zhao et al., 2020); that is, increased number of viral entry points, and thus, increased susceptibility of AT2 cells to COVID-19 infection. Furthermore, given the reciprocal interactions between LIFs and neighboring AT2 cells, in which LIFs traffic lipid substrates for surfactant production to AT2 cells (Rehan and Torday, 2014), it is highly plausible that aberrant lipid accumulation in AT2 cells and subsequent AT2 hyperplasia may also stem from impaired lipid trafficking from LIFs to AT2 cells.

In addition, obesity-associated AT2 hyperplasia may also explain the histologic features of samples derived from IPF lungs, as well as patients with severe COVID-19 cases (Mora et al., 2005; Selman and Pardo, 2006; Wigén et al., 2020), showing distinct foci of hyperplastic AT2 cells. Moreover, in obese lungs, LIFs, and AT2 cells alike, extensively accumulate lipid droplets (Foster et al., 2010), which in turn may lead to lipotoxicity, and lipotoxicity-mediated outcomes, such as cell cycle arrest and apoptosis, often observed in IPF among other lung pathologies.

FGF10-FGFR2b signaling has been shown to promote the resolution of pulmonary fibrosis via a number of mechanisms, including the one we mentioned earlier in this review (El Agha et al., 2017b). Although the precise role of FGF10 in IPF pathology remains unclear, studies found significantly elevated FGF10 expression levels in lung tissues of IPF patients compared to non-IPF donors. Strikingly, fibrotic foci, in contrast to fibrotic lesions, had detectable FGF10 protein expression, but rather at lower levels (El Agha et al., 2017a). Based on these observations, some have speculated that FGF10 is unlikely to be involved in the initial triggering events of IPF, but rather inducted to promote fibrosis resolution by counteracting the effects of TGF- $\beta 1$ (Gupte et al., 2009; El Agha et al., 2017b).

In a more recent study using bleomycin injury models, FGF10 overexpression in BESCs was shown to enhance fibrosis resolution by promoting alveolar epithelial regeneration over the development of fibrotic honeycomb lesions, while inactivation of its receptor FGFR2b led to impaired epithelial regeneration by BESCs (Yuan et al., 2019). The need for elevated FGF10 levels to induce BESCs-driven AEC regeneration and fibrosis resolution following lung injury may explain why IPF is tightly correlated with aging, as FGF10 expression is known to decrease with age. Taken together, investigating the specific role of FGF10 and its receptor FGFR2B in IPF may help create effective 
anti-fibrotic FGF10-based therapies that precisely target this signaling pathway with optimal efficacy.

Deficient FGF10 signaling during embryonic lung development is associated with BPD, a lethal lung developmental disorder of prematurely born infants. Hyperoxia injury models of BPD in Fgf 10 heterozygous pups resulted in complete postnatal lethality, starting at day 5 , while in normoxia, no postnatal lethality was observed (Chao et al., 2017). This suggests that a potential beneficial effect of recombinant FGF10 treatment is likely to be effective in the context of BPD. This could, in turn, translate into the development of novel FGF10-based therapies that may improve the prognosis of BPD in preterm neonates.

\section{CONCLUSION AND PERSPECTIVE}

By looking at the numerous developmental and functional similarities between pulmonary LIFs and adipocytes, especially white adipocytes, a few lessons have emerged. We believe that these lessons, particularly the findings pertaining to adipocyte biology, including our knowledge about FGF10 signaling in these cells, can be extrapolated to explain, at least some aspects of LIF biology in normal and diseased lungs. To achieve this, further studies using scRNA-seq and lineage tracing tools are required to further explore the influence of FGF10 signaling in LIFs, examine the role of LIF progenitors beyond early developmental stages, and better assess the functional heterogeneity of pulmonary LIFs. For instance, the newly generated Fgf10-CreERT2 line that allows lineage tracing of Fgf10-pos cells during development and postnatally may

\section{REFERENCES}

Agrati, C., Sacchi, A., Bordoni, V., Cimini, E., Notari, S., Grassi, G., et al. (2020). Expansion of myeloid-derived suppressor cells in patients with severe coronavirus disease (COVID-19). Cell Death Differ. 27, 3196-3207. doi: 10.1038/s41418-020-0572-6

Ahima, R. S., and Lazar, M. A. (2008). Adipokines and the peripheral and neural control of energy balance. Mol. Endocrinol. 22, 1023-1031. doi: 10.1210/me.2007-0529

Al Alam, D., El Agha, E., Sakurai, R., Kheirollahi, V., Moiseenko, A., Danopoulos, S., et al. (2015). Evidence for the involvement of fibroblast growth factor 10 in lipofibroblast formation during embryonic lung development. Development 142, 4139-4150. doi: 10.1242/dev.109173

Amri, E. Z., Teboul, L., Vannier, C., Grimaldi, P. A., and Ailhaud, G. (1996). Fatty acids regulate the expression of lipoprotein lipase gene and activity in preadipose and adipose cells. Biochem. J. 314, 541-546. doi: 10.1042/bj3140541

Asaki, T., Konishi, M., Miyake, A., Kato, S., Tomizawa, M., and Itoh, N. (2004). Roles of fibroblast growth factor 10 (Fgf10) in adipogenesis in vivo. Mol. Cell. Endocrinol. 218, 119-128. doi: 10.1016/j.mce.2003.12.017

Barbatelli, G., Murano, I., Madsen, L., Hao, Q., Jimenez, M., Kristiansen, K., et al. (2010). The emergence of cold-induced brown adipocytes in mouse white fat depots is determined predominantly by white to brown adipocyte transdifferentiation. Am. J. Physiol. Endocrinol. Metab. 298, E1244-E1253. doi: 10.1152/ajpendo.00600.2009

Barkauskas, C. E., Cronce, M. J., Rackley, C. R., Bowie, E. J., Keene, D. R., Stripp, B. R., et al. (2013). Type 2 alveolar cells are stem cells in adult lung. J. Clin. Invest. 123, 3025-3036. doi: 10.1172/JCI68782

Bellusci, S., Grindley, J., Emoto, H., Itoh, N., and Hogan, B. L. (1997). Fibroblast growth factor 10 (FGF10) and branching morphogenesis in the embryonic mouse lung. Development 124, 4867-4878. doi: 10.1242/dev.124.23.4867 be helpful. Such studies will contribute significantly to our understanding of the role of LIFs in lung health and disease. Also, given the importance of adipocytes and adipocyte-like cells in multiple physiological processes such as regulation of systemic energy homeostasis, and their implications for metabolic diseases such as obesity, a detailed understanding of the mechanisms by which FGF10 functions in these cells may provide profound insights into disease pathophysiology, and lead to development of novel FGF10-based therapies.

\section{AUTHOR CONTRIBUTIONS}

J-SZ and SB conceived the study and edited and revised the manuscript. Y-QL and QD drafted the manuscript and designed the figures. CC and XL provided the valuable intellectual input. All authors contributed to the article and approved the submitted version.

\section{FUNDING}

J-SZ was supported by a start-up package from Wenzhou Medical University and the Interventional Pulmonary Key Laboratory of Zhejiang Province. SB was supported by grants from the Deutsche Forschungsgemeinschaft (DFG; BE4443/6-1, KFO309 P7, and SFB CRC1213-projects A04) and the DZL. CC was supported by the National Key Research and Development Program of China (2016YFC1304000) and the Interventional Pulmonary Key Laboratory of Zhejiang Province.

Cannon, B., and Nedergaard, J. (2004). Brown adipose tissue: function and physiological significance. Physiol. Rev. 84, 277-359. doi: 10.1152/ physrev.00015.2003

Chao, C. M., Yahya, F., Moiseenko, A., Tiozzo, C., Shrestha, A., Ahmadvand, N., et al. (2017). Fgf10 deficiency is causative for lethality in a mouse model of bronchopulmonary dysplasia. J. Pathol. 241, 91-103. doi: 10.1002/path.4834

Chen, W. A. (2020). Potential treatment of COVID-19 with TGF- $\beta$ blockade. Int. J. Biol. Sci. 16, 1954-1955. doi: 10.7150/ijbs.46891

Chen, J., Wu, H., Yu, Y., and Tang, N. (2020). Pulmonary alveolar regeneration in adult COVID-19 patients. Cell Res. 30, 708-710. doi: 10.1038/s41422-0200369-7

Cinti, S. (2005). The adipose organ. Prostaglandins Leukot. Essent. Fat. Acids 73, 9-15. doi: 10.1016/j.plefa.2005.04.010

Correll, K. A., Edeen, K. E., Zemans, R. L., Redente, E. F., Serban, K. A., Curran-Everett, D., et al. (2019). Transitional human alveolar type II epithelial cells suppress extracellular matrix and growth factor gene expression in lung fibroblasts. Am. J. Phys. Lung Cell. Mol. Phys. 317, L283-1294. doi: 10.1152/ajplung.00337.2018

De Moerlooze, L., Spencer-Dene, B., Revest, J. M., Hajihosseini, M., Rosewell, I., and Dickson, C. (2000). An important role for the IIIb isoform of fibroblast growth factor receptor 2 (FGFR2) in mesenchymal-epithelial signalling during mouse organogenesis. Development 127, 483-492. doi: 10.1242/dev.127.3.483

Dutchak, P. A., Katafuchi, T., Bookout, A. L., Choi, J. H., Yu, R. T., Mangelsdorf, D. J., et al. (2012). Fibroblast growth factor-21 regulates PPAR $\gamma$ activity and the antidiabetic actions of thiazolidinediones. Cell 148, 556-567. doi: 10.1016/j. cell.2011.11.062

El Agha, E., and Bellusci, S. (2014). Walking along the fibroblast growth factor 10 route: a key pathway to understand the control and regulation of epithelial and mesenchymal cell-lineage formation during lung development and repair after injury. Scientifica 2014:538379. doi: 10.1155/2014/538379 
El Agha, E., Herold, S., Al Alam, D., Quantius, J., MacKenzie, B., Carraro, G., et al. (2014). Fgf10-positive cells represent a progenitor cell population during lung development and postnatally. Development 141, 296-306. doi: 10.1242/dev.099747

El Agha, E., Kramann, R., Schneider, R. K., Li, X., Seeger, W., Humphreys, B. D., et al. (2017a). Mesenchymal stem cells in fibrotic disease. Cell Stem Cell 21, 166-177. doi: 10.1016/j.stem.2017.07.011

El Agha, E., Moiseenko, A., Kheirollahi, V., De Langhe, S., Crnkovic, S., Kwapiszewska, G., et al. (2017b). Two-way conversion between lipogenic and myogenic fibroblastic phenotypes marks the progression and resolution of lung fibrosis. Cell Stem Cell 20, 261-273.e263. doi: 10.1016/j.stem. 2016.10.004

Ferreira-Gomes, M., Kruglov, A., Durek, P., Heinrich, F., Tizian, C., Anne, H. G., et al. (2021). SARS-CoV-2 in severe COVID-19 induces a TGF- $\beta$-dominated chronic immune response that does not target itself. Nat. Commun. 12:1961. doi: 10.1038/s41467-021-22210-3

Fischer, C., Seki, T., Lim, S., Nakamura, M., Andersson, P., Yang, Y., et al. (2017). A miR-327-FGF10-FGFR2-mediated autocrine signaling mechanism controls white fat browning. Nat. Commun. 8:2079. doi: 10.1038/s41467-01702158-Z

Fisher, F. M., Kleiner, S., Douris, N., Fox, E. C., Mepani, R. J., Verdeguer, F., et al. (2012). FGF21 regulates PGC-1 $\alpha$ and browning of white adipose tissues in adaptive thermogenesis. Genes Dev. 26, 271-281. doi: 10.1101/ gad.177857.111

Foster, D. J., Ravikumar, P., Bellotto, D. J., Unger, R. H., and Hsia, C. C. (2010). Fatty diabetic lung: altered alveolar structure and surfactant protein expression. Am. J. Phys. Lung Cell. Mol. Phys. 298, L392-L403. doi: 10.1152/ ajplung.00041.2009

Fux, C., Mitta, B., Kramer, B. P., and Fussenegger, M. (2004). Dual-regulated expression of C/EBP-alpha and BMP-2 enables differential differentiation of $\mathrm{C} 2 \mathrm{C} 12$ cells into adipocytes and osteoblasts. Nucleic Acids Res. 32:e1. doi: 10.1093/nar/gnh001

Gartside, M. G., Chen, H., Ibrahimi, O. A., Byron, S. A., Curtis, A. V., Wellens, C. L., et al. (2009). Loss-of-function fibroblast growth factor receptor-2 mutations in melanoma. Mol. Cancer Res. 7, 41-54. doi: 10.1158/1541-7786. Mcr-08-0021

Gries, C. J., Bhadriraju, S., Edelman, J. D., Goss, C. H., Raghu, G., and Mulligan, M. S. (2015). Obese patients with idiopathic pulmonary fibrosis have a higher 90-day mortality risk with bilateral lung transplantation. J. Heart Lung Transplant. 34, 241-246. doi: 10.1016/j.healun.2014.09.031

Gupte, V. V., Ramasamy, S. K., Reddy, R., Lee, J., Weinreb, P. H., Violette, S. M., et al. (2009). Overexpression of fibroblast growth factor-10 during both inflammatory and fibrotic phases attenuates bleomycin-induced pulmonary fibrosis in mice. Am. J. Respir. Crit. Care Med. 180, 424-436. doi: 10.1164/ rccm.200811-1794OC

Hinz, B., Phan, S. H., Thannickal, V. J., Prunotto, M., Desmoulière, A., Varga, J., et al. (2012). Recent developments in myofibroblast biology: paradigms for connective tissue remodeling. Am. J. Pathol. 180, 1340-1355. doi: 10.1016/j. ajpath.2012.02.004

Hsieh, I. N., De Luna, X., White, M. R., and Hartshorn, K. L. (2018). The role and molecular mechanism of action of surfactant protein $\mathrm{D}$ in innate host defense against influenza A virus. Front. Immunol. 9:1368. doi: 10.3389/ fimmu.2018.01368

Huang, H., Song, T. J., Li, X., Hu, L., He, Q., Liu, M., et al. (2009). BMP signaling pathway is required for commitment of C3H10T1/2 pluripotent stem cells to the adipocyte lineage. Proc. Natl. Acad. Sci. U. S. A. 106, 12670-12675. doi: 10.1073/pnas.0906266106

Inselman, L. S., Chander, A., and Spitzer, A. R. (2004). Diminished lung compliance and elevated surfactant lipids and proteins in nutritionally obese young rats. Lung 182, 101-117. doi: 10.1007/s00408-003-1048-4

Kersten, S., Desvergne, B., and Wahli, W. (2000). Roles of PPARs in health and disease. Nature 405, 421-424. doi: 10.1038/35013000

Kim, K. K., Kugler, M. C., Wolters, P. J., Robillard, L., Galvez, M. G., Brumwell, A. N., et al. (2006). Alveolar epithelial cell mesenchymal transition develops in vivo during pulmonary fibrosis and is regulated by the extracellular matrix. Proc. Natl. Acad. Sci. U. S. A. 103, 13180-13185. doi: 10.1073/ pnas.0605669103

King, T. E. Jr., Pardo, A., and Selman, M. (2011). Idiopathic pulmonary fibrosis. Lancet 378, 1949-1961. doi: 10.1016/S0140-6736(11)60052-4
Kis, K., Liu, X., and Hagood, J. S. (2011). Myofibroblast differentiation and survival in fibrotic disease. Expert Rev. Mol. Med. 13:e27. doi: 10.1017/ S1462399411001967

Klingberg, F., Hinz, B., and White, E. S. (2013). The myofibroblast matrix: implications for tissue repair and fibrosis. J. Pathol. 229, 298-309. doi: 10.1002/path.4104

Konishi, M., Asaki, T., Koike, N., Miwa, H., Miyake, A., and Itoh, N. (2006). Role of Fgf10 in cell proliferation in white adipose tissue. Mol. Cell. Endocrinol. 249, 71-77. doi: 10.1016/j.mce.2006.01.010

Kruglikov, I. L., and Scherer, P. E. (2020). The role of adipocytes and adipocytelike cells in the severity of COVID-19 infections. Obesity (Silver Spring) 28, 1187-1190. doi: 10.1002/oby.22856

Lane, M. D., Tang, Q. Q., and Jiang, M. S. (1999). Role of the CCAAT enhancer binding proteins (C/EBPs) in adipocyte differentiation. Biochem. Biophys. Res. Commun. 266, 677-683. doi: 10.1006/bbrc.1999.1885

Lowell, B. B., and Flier, J. S. (1997). Brown adipose tissue, beta 3-adrenergic receptors, and obesity. Annu. Rev. Med. 48, 307-316. doi: 10.1146/annurev. med.48.1.307

Mason, R. J. (2006). Biology of alveolar type II cells. Respirology 11, S12-S15. doi: $10.1111 / \mathrm{j} .1440-1843.2006 .00800 . x$

Mead, J. R., Irvine, S. A., and Ramji, D. P. (2002). Lipoprotein lipase: structure, function, regulation, and role in disease. J. Mol. Med. 80, 753-769. doi: 10.1007/s00109-002-0384-9

Min, H., Danilenko, D. M., Scully, S. A., Bolon, B., Ring, B. D., Tarpley, J. E., et al. (1998). Fgf-10 is required for both limb and lung development and exhibits striking functional similarity to Drosophila branchless. Genes Dev. 12, 3156-3161. doi: 10.1101/gad.12.20.3156

Mora, A. L., Woods, C. R., Garcia, A., Xu, J., Rojas, M., Speck, S. H., et al. (2005). Lung infection with gamma-herpesvirus induces progressive pulmonary fibrosis in Th2-biased mice. Am. J. Phys. Lung Cell. Mol. Phys. 289, L711-L721. doi: 10.1152/ajplung.00007.2005

Nathan, S. D., Shlobin, O. A., Ahmad, S., Burton, N. A., Barnett, S. D., and Edwards, E. (2010). Comparison of wait times and mortality for idiopathic pulmonary fibrosis patients listed for single or bilateral lung transplantation. J. Heart Lung Transplant. 29, 1165-1171. doi: 10.1016/j.healun.2010. 05.014

Ndlovu, R., Deng, L. C., Wu, J., Li, X. K., and Zhang, J. S. (2018). Fibroblast growth factor 10 in pancreas development and pancreatic cancer. Front. Genet. 9:482. doi: 10.3389/fgene.2018.00482

Ohta, H., and Itoh, N. (2014). Roles of FGFs as adipokines in adipose tissue development, remodeling, and metabolism. Front. Endocrinol. 5:18. doi: $10.3389 /$ fendo. 2014.00018

Ohta, H., Konishi, M., and Itoh, N. (2011). FGF10 and FGF21 as regulators in adipocyte development and metabolism. Endocr Metab Immune Disord Drug Targets 11, 302-309. doi: 10.2174/187153011797881166

Ohuchi, H., Hori, Y., Yamasaki, M., Harada, H., Sekine, K., Kato, S., et al. (2000). FGF10 acts as a major ligand for FGF receptor 2 IIIb in mouse multi-organ development. Biochem. Biophys. Res. Commun. 277, 643-649. doi: 10.1006/bbrc.2000.3721

Ornitz, D. M., and Itoh, N. (2015). The fibroblast growth factor signaling pathway. Wiley Interdiscip. Rev. Dev. Biol. 4, 215-266. doi: 10.1002/ wdev.176

Otto, T. C., Bowers, R. R., and Lane, M. D. (2007). BMP-4 treatment of C3H10T1/2 stem cells blocks expression of MMP-3 and MMP-13. Biochem. Biophys. Res. Commun. 353, 1097-1104. doi: 10.1016/j.bbrc.2006. 12.170

Patel, N. G., Kumar, S., and Eggo, M. C. (2005). Essential role of fibroblast growth factor signaling in preadipoctye differentiation. J. Clin. Endocrinol. Metab. 90, 1226-1232. doi: 10.1210/jc.2004-1309

Pearson, R., Fleetwood, J., Eaton, S., Crossley, M., and Bao, S. (2008). Krüppellike transcription factors: a functional family. Int. J. Biochem. Cell Biol. 40, 1996-2001. doi: 10.1016/j.biocel.2007.07.018

Pei, H., Yao, Y., Yang, Y., Liao, K., and Wu, J. R. (2011). Krüppel-like factor KLF9 regulates PPAR $\gamma$ transactivation at the middle stage of adipogenesis Cell Death Differ. 18, 315-327. doi: 10.1038/cdd.2010.100

Rehan, V. K., and Torday, J. S. (2014). The lung alveolar lipofibroblast: an evolutionary strategy against neonatal hyperoxic lung injury. Antioxid. Redox Signal. 21, 1893-1904. doi: 10.1089/ars.2013.5793

Richard, D., and Picard, F. (2011). Brown fat biology and thermogenesis. Front. Biosci. 16, 1233-1260. doi: 10.2741/3786 
Rosen, E. D., and MacDougald, O. A. (2006). Adipocyte differentiation from the inside out. Nat. Rev. Mol. Cell Biol. 7, 885-896. doi: 10.1038/ nrm2066

Rosen, E. D., and Spiegelman, B. M. (2000). Molecular regulation of adipogenesis. Annu. Rev. Cell Dev. Biol. 16, 145-171. doi: 10.1146/annurev. cellbio.16.1.145

Sakaue, H., Konishi, M., Ogawa, W., Asaki, T., Mori, T., Yamasaki, M., et al. (2002). Requirement of fibroblast growth factor 10 in development of white adipose tissue. Genes Dev. 16, 908-912. doi: 10.1101/gad.983202

Sanchez-Gurmaches, J., and Guertin, D. A. (2014). Adipocytes arise from multiple lineages that are heterogeneously and dynamically distributed. Nat. Commun. 5:4099. doi: $10.1038 /$ ncomms5099

Sanchez-Gurmaches, J., Hung, C. M., and Guertin, D. A. (2016). Emerging complexities in adipocyte origins and identity. Trends Cell Biol. 26, 313-326. doi: 10.1016/j.tcb.2016.01.004

Schmidt, R., Meier, U., Markart, P., Grimminger, F., Velcovsky, H. G., Morr, H., et al. (2002). Altered fatty acid composition of lung surfactant phospholipids in interstitial lung disease. Am. J. Phys. Lung Cell. Mol. Phys. 283, L1079-L1085. doi: 10.1152/ajplung.00484.2001

Schoonjans, K., Staels, B., and Auwerx, J. (1996). The peroxisome proliferator activated receptors (PPARS) and their effects on lipid metabolism and adipocyte differentiation. Biochim. Biophys. Acta 1302, 93-109. doi: 10.1016/ 0005-2760(96)00066-5

Schulz, T. J., Huang, T. L., Tran, T. T., Zhang, H., Townsend, K. L., Shadrach, J. L., et al. (2011). Identification of inducible brown adipocyte progenitors residing in skeletal muscle and white fat. Proc. Natl. Acad. Sci. U. S. A. 108, 143-148. doi: $10.1073 /$ pnas. 1010929108

Sebo, Z. L., Jeffery, E., Holtrup, B., and Rodeheffer, M. S. (2018). A mesodermal fate map for adipose tissue. Development 145:dev166801. doi: 10.1242/ dev.166801

Selman, M., and Pardo, A. (2006). Role of epithelial cells in idiopathic pulmonary fibrosis: from innocent targets to serial killers. Proc. Am. Thorac. Soc. 3 , 364-372. doi: 10.1513/pats.200601-003TK

Sime, P. J., and O'Reilly, K. M. (2001). Fibrosis of the lung and other tissues: new concepts in pathogenesis and treatment. Clin. Immunol. 99, 308-319. doi: $10.1006 /$ clim.2001.5008

Steele, M. P., and Schwartz, D. A. (2013). Molecular mechanisms in progressive idiopathic pulmonary fibrosis. Annu. Rev. Med. 64, 265-276. doi: 10.1146/ annurev-med-042711-142004

Sun, Y., Wang, R., Zhao, S., Li, W., Liu, W., Tang, L., et al. (2019). FGF9 inhibits browning program of white adipocytes and associates with human obesity. J. Mol. Endocrinol. 62, 79-90. doi: 10.1530/JME-18-0151
Tomasek, J. J., Gabbiani, G., Hinz, B., Chaponnier, C., and Brown, R. A. (2002). Myofibroblasts and mechano-regulation of connective tissue remodelling. Nat. Rev. Mol. Cell Biol. 3, 349-363. doi: 10.1038/nrm809

Tordet, C., Marin, L., and Dameron, F. (1981). Pulmonary di-and-triacylglycerols during the perinatal development of the rat. Experientia 37, 333-334. doi: 10.1007/BF01959845

Weaver, M., Dunn, N. R., and Hogan, B. L. (2000). Bmp4 and Fgf10 play opposing roles during lung bud morphogenesis. Development 127, 2695-2704. doi: $10.1242 /$ dev.127.12.2695

Wigén, J., Löfdahl, A., Bjermer, L., Elowsson-Rendin, L., and Westergren-Thorsson, G. (2020). Converging pathways in pulmonary fibrosis and Covid-19 - The fibrotic link to disease severity. Respir. Med. X 2:100023. doi: 10.1016/j.yrmex.2020.100023

Wu, J., Boström, P., Sparks, L. M., Ye, L., Choi, J. H., Giang, A. H., et al. (2012). Beige adipocytes are a distinct type of thermogenic fat cell in mouse and human. Cell 150, 366-376. doi: 10.1016/j.cell.2012.05.016

Wu, Z., and Wang, S. (2013). Role of kruppel-like transcription factors in adipogenesis. Dev. Biol. 373, 235-243. doi: 10.1016/j.ydbio.2012.10.031

$\mathrm{Xu}$, Q., Lin, S., Wangsid, Y., Zhu, J., and Lin, Y. (2018). Fibroblast growth factor 10 (FGF10) promotes the adipogenesis of intramuscular preadipocytes in goat. Mol. Biol. Rep. 45, 1881-1888. doi: 10.1007/s11033-018-4334-1

Yamasaki, M., Emoto, H., Konishi, M., Mikami, T., Ohuchi, H., Nakao, K., et al. (1999). FGF-10 is a growth factor for preadipocytes in white adipose tissue. Biochem. Biophys. Res. Commun. 258, 109-112. doi: 10.1006/bbrc.1999.0594

Yuan, T., Volckaert, T., Redente, E. F., Hopkins, S., Klinkhammer, K., Wasnick, R., et al. (2019). FGF10-FGFR2B signaling generates basal cells and drives alveolar epithelial regeneration by bronchial epithelial stem cells after lung injury. Stem Cell Rep. 12, 1041-1055. doi: 10.1016/j.stemcr.2019.04.003

Zhao, Y., Zhao, Z., Wang, Y., Zhou, Y., Ma, Y., and Zuo, W. (2020). Single-cell RNA expression profiling of ACE2, the receptor of SARS-CoV-2. Am. J. Respir. Crit. Care Med. 202, 756-759. doi: 10.1164/rccm.202001-0179LE

Conflict of Interest: The authors declare that the research was conducted in the absence of any commercial or financial relationships that could be construed as a potential conflict of interest.

Copyright (c) 2021 Lv, Dhlamini, Chen, Li, Bellusci and Zhang. This is an open-access article distributed under the terms of the Creative Commons Attribution License (CC BY). The use, distribution or reproduction in other forums is permitted, provided the original author(s) and the copyright owner(s) are credited and that the original publication in this journal is cited, in accordance with accepted academic practice. No use, distribution or reproduction is permitted which does not comply with these terms. 